\title{
Significant Growth Inhibition of Canine Mammary Carcinoma Xenografts following Treatment with Oncolytic Vaccinia Virus GLV-1h68
}

\author{
Ivaylo Gentschev, ${ }^{1,2}$ Klaas Ehrig, ${ }^{2}$ Ulrike Donat, ${ }^{2}$ Michael Hess, ${ }^{2}$ Stephan Rudolph, ${ }^{2}$ \\ Nanhai Chen, ${ }^{1}$ Yong A. Yu, ${ }^{1}$ Qian Zhang, ${ }^{1}$ Jörn Bullerdiek, ${ }^{3,4}$ Ingo Nolte, ${ }^{4}$ Jochen Stritzker, ${ }^{1,2}$ \\ and Aladar A. Szalay $1,2,5,6,7$
}

${ }^{1}$ Genelux Corporation, San Diego Science Center, San Diego, CA 92109, USA

${ }^{2}$ Department of Biochemistry, University of Wuerzburg, 97074 Wuerzburg, Germany

${ }^{3}$ Center for Human Genetics, University of Bremen, 28359 Bremen, Germany

${ }^{4}$ Small Animal Clinic, University of Veterinary Medicine, Bischofsholer Damm 15, 30173 Hannover, Germany

${ }^{5}$ Rudolf Virchow Center for Experimental Biomedicine, University of Wuerzburg, 97078 Wuerzburg, Germany

${ }^{6}$ Institute for Molecular Infection Biology, University of Wuerzburg, 97078 Wuerzburg, Germany

${ }^{7}$ Department of Radiation Oncology, Moores Cancer Center, University of California, San Diego, 3855 Health Sciences Drive 0843, La Jolla, CA 92093-0843, USA

Correspondence should be addressed to Aladar A. Szalay, aaszalay@genelux.com

Received 28 August 2009; Revised 18 February 2010; Accepted 11 May 2010

Academic Editor: Dominic Fan

Copyright ( $) 2010$ Ivaylo Gentschev et al. This is an open access article distributed under the Creative Commons Attribution License, which permits unrestricted use, distribution, and reproduction in any medium, provided the original work is properly cited.

\begin{abstract}
Canine mammary carcinoma is a highly metastatic tumor that is poorly responsive to available treatment. Therefore, there is an urgent need to identify novel agents for therapy of this disease. Recently, we reported that the oncolytic vaccinia virus GLV-1h68 could be a useful tool for therapy of canine mammary adenoma in vivo. In this study we analyzed the therapeutic effect of GLV1 h68 against canine mammary carcinoma. Cell culture data demonstrated that GLV-1h68 efficiently infected and destroyed cells of the mammary carcinoma cell line MTH52c. Furthermore, after systemic administration, this attenuated vaccinia virus strain primarily replicated in canine tumor xenografts in nude mice. Finally, infection with GLV-1h68 led to strong inflammatory and oncolytic effects resulting in significant growth inhibition of the tumors. In summary, the data showed that the GLV-1h68 virus strain has promising potential for effective treatment of canine mammary carcinoma.
\end{abstract}

\section{Introduction}

Malignant tumors of the mammary glands are among the most frequently observed tumors in female dogs [1-3]. Despite the success in diagnosis and treatment of mammary cancer, this disease entity remains one of the leading causes of cancer-related death in female dogs. Therefore, there is an urgent need to identify novel agents for therapy and diagnosis of mammary cancer. Among the most promising new therapeutic candidates are oncolytic viruses, which can target tumor tissue and specifically eradicate the cancer cells. This concept was already confirmed in human tumor xenograft treatment by the use of several viruses [4-9].

In the present study, we tested the recombinant oncolytic vaccinia virus GLV- $1 \mathrm{~h} 68$ as a therapeutic agent against canine mammary carcinoma. The GLV-1h68 virus strain was engineered by inserting expression cassettes encoding a Renilla luciferase-green fluorescent protein (GFP) fusion protein, $\beta$ galactosidase, and $\beta$-glucuronidase into the genome of the wild-type strain LIVP [10]. In nude mouse models, GLV1 h68 is highly attenuated compared to the wild-type strain [10]. We have already demonstrated that the injection of 
GLV-1h68 leads to regression and elimination of different tumor xenografts in nude mice [10-15]. More recently, we reported that GLV-1h68 could be a useful tool for therapy of canine mammary adenoma [12].

Here we describe that the GLV-1h68 virus successfully infected, replicated, and lysed canine mammary carcinoma MTH52c cells in cell culture. In addition, GLV-1h68 can efficiently prevent cancer growth in female nude mice with tumors derived from MTH52c cells. Finally, the localization and effects of GLV-1h68 in the primary tumor were analyzed by immunohistochemical studies and by mouse ImmuneRelated Protein Antigen Profiling.

\section{Materials and Methods}

2.1. Cell Culture. African green monkey kidney fibroblasts $(\mathrm{CV}-1)$ were obtained from the American Type Culture Collection (ATCC). MTH52c is derived from a malignant small-cell canine carcinoma [16].

Cells were cultured in DMEM supplemented with antibiotic solution $(100 \mathrm{U} / \mathrm{ml}$ penicillin $\mathrm{G}, 100$ units $/ \mathrm{ml}$ streptomycin) and 10\% fetal bovine serum (FBS; Invitrogen $\mathrm{GmbH}$, Karlsruhe, Germany) for CV-1 and 20\% FBS for MTH52c at $37^{\circ} \mathrm{C}$ under $5 \% \mathrm{CO}_{2}$.

2.2. Virus Strain. GLV-1h68 is a genetically stable oncolytic virus strain designed to locate, enter, colonize, and destroy cancer cells without harming healthy tissues or organs [10].

2.3. Cell Viability Assay with GLV-1h68. MTH52c cells were seeded in 24-well plates (Nunc, Wiesbaden, Germany). After 24 hours in culture, cells were infected with GLV-1h68 using multiplicities of infection (MOI) of 0.1 and 1.0. Cells were incubated at $37^{\circ} \mathrm{C}$ for 1 hour, after which the infection medium was removed, and cells were subsequently incubated in fresh growth medium. The amount of viable cells after infection with GLV-1h68 was measured as described previously [12].

2.4. Viral Replication. For the viral replication assay, MTH52c cells grown in 24-well plates were infected with GLV-1h68 at an MOI of 0.1. After one hour of incubation at $37^{\circ} \mathrm{C}$ with gentle agitation every 20 minutes, the infection medium was removed and replaced by a fresh growth medium. After 1, 6, 12, 24, 48, 72, and 96 hours, the cells and supernatants were harvested. Following three freeze-thaw cycles, serial dilutions of the lysates were titered by standard plaque assays on CV-1 cells. All samples were measured in triplicate.

2.5. Western Blot Analysis of Virus-Mediated Marker Proteins. Three days prior to infection, MTH52c cells were seeded in 24-well plates (Nunc, Wiesbaden, Germany). If not otherwise indicated, the $90 \%$ confluent cell layer was mockinfected or infected with GLV-1h68 at MOIs of 0.1 and 1.0 for 1 hour at $37^{\circ} \mathrm{C}$. The virus-containing medium was aspirated and replaced by fresh medium containing $20 \%$ FBS. For protein isolation and detection, cells were harvested and resuspended in sodium dodecyl sulfate (SDS) sample buffer at one, 12, 24, 48, 72, and 96 hours post infection (hpi). The protein samples were separated by $10 \%$ SDS polyacrylamide gel electrophoresis (PAGE) and subsequently blotted onto a nitrocellulose membrane (Whatman $\mathrm{GmbH}$, Dassel, Germany). The membrane was then incubated with anti-beta-actin mouse monoclonal antibodies (ab6276, Abcam, Cambridge, UK), anti-beta-galactosidase rabbit polyclonal antibodies (A-11132, Molecular Probes, Leiden, Netherlands), or anti-GFP rabbit polyclonal antibodies (sc8334, Santa Cruz, Heidelberg, Germany), and detection was obtained using horseradish peroxidise-labeled secondary antibodies against mice (ab6728, Abcam, Cambridge, UK) or rabbits (ab6721, Abcam, Cambridge, UK) followed by enhanced chemiluminescence.

2.6. Fluorescence Imaging. The GFP signals of virus-infected cells and animals were analyzed with a fluorescence microscope (Leica DM IRB; Wetzlar, Germany) and a fluorescence stereomicroscope (Leica MZ 16 FA; Wetzlar, Germany), respectively. Images were captured with an electronic camera and were processed using META-MORPH (Universal Imaging; Downingtown, PA, USA) and Photoshop 7.0 (Adobe Systems, Mountain View, CA, USA).

2.7. Flow Cytometry (FACS) Analysis. MTH52c cells were grown on 24-well plates (Nunc, Wiesbaden, Germany) and infected by GLV-1h68 at an MOI of 0.1 or 1.0, respectively. At various time points, infected and noninfected MTH52c cells were harvested by Trypsin-EDTA treatment (PAA Laboratories $\mathrm{GmbH}$, Pasching, Austria) and resuspended in PBS. For discrimination between viable and dead, MTH52c cells were stained using $2 \mu \mathrm{l}$ propidium iodide $(1 \mathrm{mg} / \mathrm{ml}$; Sigma, Taufkirchen, Germany) per $1 \mathrm{ml}$ cell suspension for 5 min at room temperature. A minimum of $2 \times 10^{5}$ cells were then measured using an Epics XL flow cytometer (Beckman Coulter GmbH Krefeld, Germany).

2.8. Bioluminescence Imaging. For monitoring studies of the distribution of the GLV-1h68 virus in tumor-bearing mice, animals were analyzed for the presence of virus-dependent luciferase activity. For this purpose, mice were injected intravenously with a mixture of $5 \mu \mathrm{l}$ of coelenterazine (Sigma, Taufkirchen, Germany; $0.5 \mu \mathrm{g} / \mu \mathrm{l}$ diluted ethanol solution) and $95 \mu \mathrm{l}$ of luciferase assay buffer $(0.5 \mathrm{M} \mathrm{NaCl} ; 1 \mathrm{mM}$ EDTA; 0.1 M potassium phosphate, $\mathrm{pH}$ 7.4). The animals were then anaesthetized with 2.5\% Isoflurane (Forene, Abbott, Ludwigshafen, Germany) in a knockout box and were maintained in an anaesthesia module aerated with $1.5 \%$ Isoflurane/oxygen. The mice were imaged using the CCD-Camera-based NightOWL LB 981 Imaging System (Berthold Technologies, Bad Wildbad, Germany). Photons were collected for 5 minutes from dorsal views of the animals, and the images were recorded using Image WinLight 32 software (Berthold Technologies, Bad Wildbad, Germany).

2.9. GLV-1h68-Mediated Therapy of MTH52c Xenografts. Tumors were generated by $5 \times 10^{6}$ implanting cells in $100 \mu \mathrm{l}$ 
PBS subcutaneously into the right hind leg of 6- to 8-weekold female nude mice (NCI/Hsd/Athymic Nude-Foxn $1^{\text {nu }}$, Harlan Winkelmann GmbH, Borchen, Germany). Tumor growth was monitored 3 times weekly in two dimensions using a digital caliper. Tumor volume was calculated as $\left[\left(\right.\right.$ length $\times$ width $\left.\left.^{2}\right) / 2\right]$. On day 12 , a single dose of GLV-1h68 virus $\left(5 \times 10^{6}\right.$ plaque forming units [pfu] in $100 \mu \mathrm{l}$ PBS $)$ was injected into the tail vein (i.v.). The control animals were injected i.v. with PBS only.

The significance of the results was calculated by two-way analysis of variance (ANOVA) using the GraphPad Prism software (San Diego, USA). Results are displayed as means \pm s.d. (standard deviation). $P$-values of $<.05$ were considered significant.

The animals were euthanized by cervical dislocation. All animal experiments were approved by the government of Unterfranken and conducted according to the German animal protection guidelines.

2.10. Toxicity Studies. Mice with MTH52c xenograft tumors were developed to assess the biodistribution and toxicity of the GLV-1h68 virus. After virus infection, animals were observed daily for any sign of toxicity, and body weight was checked twice weekly. At day 21 and 42 after injection, viral distribution in animals from each group was analyzed. The tumors and organs were excised, inspected, and homogenized using FastPrep FP120 Cell Disruptor (BIO 101, Qbiogene, Germany) at a speed of 6 for 20 s (three times). After three freeze-thaw cycles, the supernatants were collected by centrifugation at $1000 \times \mathrm{g}$ for 5 minutes. The viral titers were then determined in duplicate by standard plaque assays using CV-1 cells.

2.11. Histological Analysis of Tumors. For histological studies, tumors were excised and snap-frozen in liquid $\mathrm{N}_{2}$, followed by fixation in $4 \%$ paraformaldehyde/PBS at $\mathrm{pH} 7.4$ for 16 $\mathrm{h}$ at $4^{\circ} \mathrm{C}$. Tissue sectioning was performed as described by Weibel et al. [17]. GLV-1h68 was labeled using polyclonal rabbit antivaccinia virus (anti-VACV) antibody (Abcam, Cambridge, UK), which was stained using Cy3-conjugated donkey antirabbit secondary antibodies obtained from Jackson ImmunoResearch (West Grove, PA, USA). PhalloidinTRITC (Sigma, Taufkirchen, Germany) was used to label actin.

The fluorescent-labeled preparations were examined using the Leica MZ 16 FA Stereo-Fluorescence microscope equipped with a Leica DC500 Digital Camera. Digital images were processed with Photoshop 7.0 (Adobe Systems, Mountain View, CA, USA) and merged to yield pseudocolored images.

2.12. Preparation of Tumor Lysates for Mouse ImmuneRelated Protein Antigen Profiling. GLV-1h68-infected and noninfected tumors of MTH52c or ZMTH3 xenografted nude mice were used for preparation of tumor lysates at 42 days after virus infection. Tumors were resuspended in 9 volumes (W/V) lysis buffer [50 mM Tris- $\mathrm{HCl}(\mathrm{pH} 7.4)$, $2 \mathrm{mM}$ EDTA (pH 7.4), $2 \mathrm{mM}$ PMSF and Complete Mini
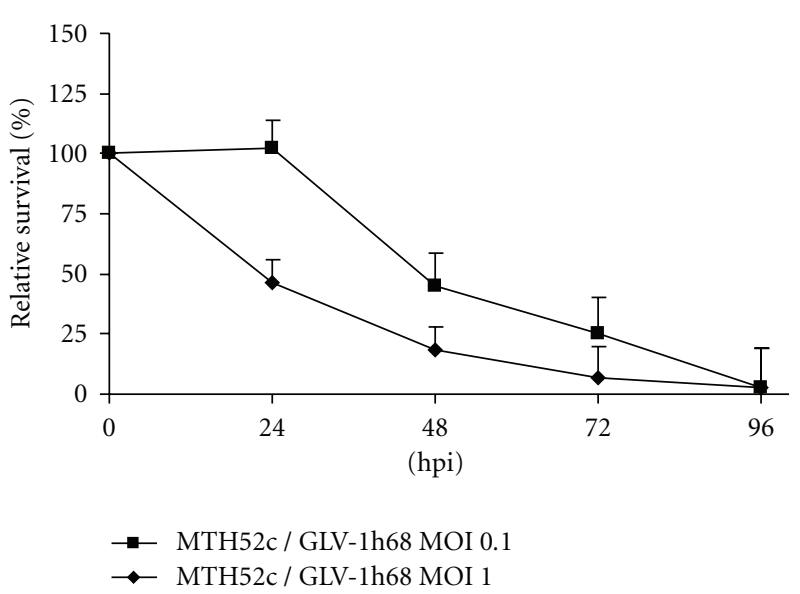

(a)

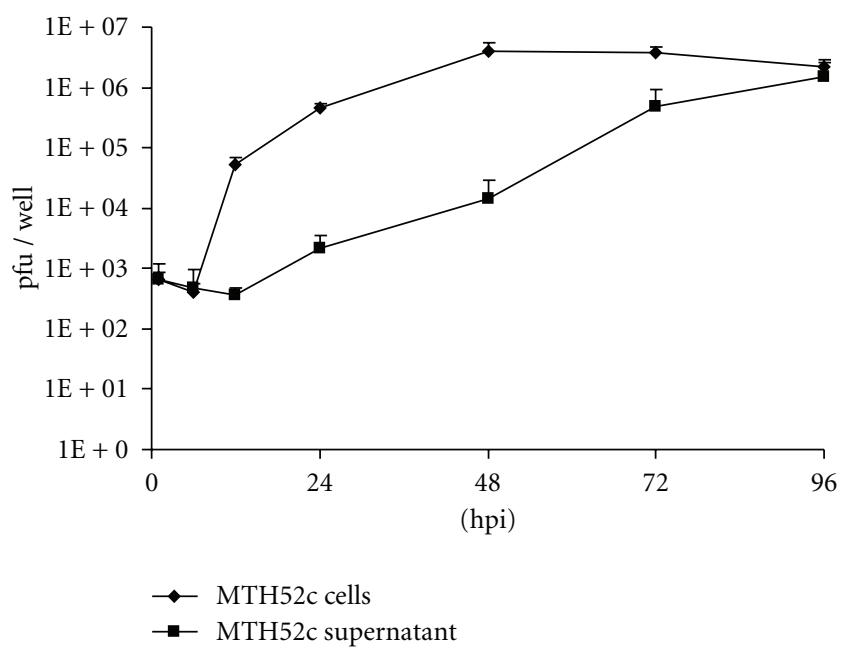

(b)

FIGURE 1: Cytotoxicity (a) and replication (b) of the GLV-1h68 virus in canine mammary MTH52c cells. (a) Viability of MTH52c cells after GLV-1h68 infection using MOIs of 0.1 and 1 was monitored over 96 hours. The amount of viable cells after infection with GLV1 h68 was measured in triplicate. Values are shown as percentages of respective uninfected controls. (b) Viral titer analysis in MTH52c cell culture after infection with GLV-1h68 at an MOI of 0.1. Cells and supernatant of virally treated cells were collected at various times post infection. Viral titers were determined as pfu per well in triplicate by plaque assay in CV-1 cell monolayers. Average plus standard deviations plotted.

protease inhibitors (Roche, Mannheim, Germany)] and lysed using a FastPrep FP120 Cell Disruptor (BIO 101, Qbiogene, Germany) at a speed of 6.0 for $20 \mathrm{~s}$ (three times). Samples were centrifuged at $20,000 \mathrm{~g}$ at $4^{\circ} \mathrm{C}$ for 5 minutes, and supernatants were then analyzed for mouse immune-related protein antigen profiling by Multianalyte Profiles (mouse MAPs; Rules-Based Medicine, Austin, USA) using antibodylinked beads. Results were normalized based on total protein concentration. 
TABLE 1: Viral titer in tissue samples (pfu/organ or tumor).

\begin{tabular}{|c|c|c|c|c|c|}
\hline Animal no./dpi & $1 / 21 \mathrm{dpi}$ & $2 / 21 \mathrm{dpi}$ & $3 / 21 \mathrm{dpi}$ & 4/42 dpi & $5 / 42 \mathrm{dpi}$ \\
\hline Liver & 120 & 1716 & 840 & n.d & n.d \\
\hline Lungs & 1520 & 480 & n.d. & 56 & 324 \\
\hline Kidneys & n.d. & n.d. & n.d. & NT & NT \\
\hline Spleen & 150 & n.d & n.d & n.d & n.d \\
\hline Ovaries & n.d. & n.d & 30 & NT & NT \\
\hline Tumor & $2 \times 10^{7}$ & $1 \times 10^{7}$ & $2.1 \times 10^{7}$ & $7.3 \times 10^{5}$ & $7.3 \times 10^{5}$ \\
\hline
\end{tabular}

Tumor-bearing mice were injected with $5 \times 10^{6}$ pfu of GLV-1h68. Mice were sacrificed at day 21 or 42 after virus injection (dpi). The data were determined by standard plaque assays on CV-1 cells using aliquots of the homogenized organs and were displayed as mean pfu/organ or tissue. For each organ, two aliquots of $0.1 \mathrm{ml}$ were measured in triplicates.

n.d.: not detected (detection LIMIT $<10$ pfu/organ).

NT: not tested.

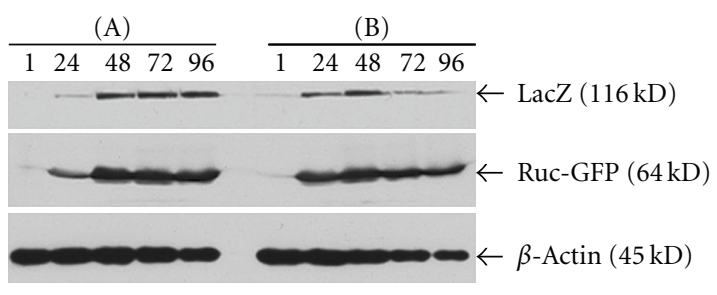

FIGURE 2: Western blot analysis of virus-mediated expression of Renilla luciferase-GFP fusion protein and $\beta$-galactosidase. MTH52c cells infected with GLV-1h68 at MOIs of 0.1 (a) and 1.0 (b) were used for protein isolation at 1, 12, 24, 48, 72, and 96 hours post infection (hpi). The time-dependent expression of Renilla luciferase-GFP fusion protein (Ruc-GFP), $\beta$-galactosidase (LacZ), and beta-actin as a control was analyzed as described in Materials and Methods.

\section{Results}

3.1. Infection and Replication of GLV-1h68 in the Canine Mammary Cell Line MTH52c. In order to test the ability of the GLV-1h68 virus to infect and lyse MTH52c cells in cell culture, we first performed a cell viability assay, as described in Materials and Methods. Ninety-six hours after GLV-1h68 infection at an MOI of 0.1 and 1.0, the MTH52c cells were eradicated, with only $2.4 \pm 1.04 \%$ and $206 \pm$ $0.68 \%$ surviving the treatment, respectively (Figure $1(\mathrm{a})$ ). These results indicate that GLV-1h68 virus infection leads to an efficient eradication of the carcinoma MTH52c cells in culture.

To determine the replication efficacy of GLV-1h68 in MTH52c cells, we analyzed both the supernatant and the cell-associated virus titers at different times post infection (Figure 1(b)). Whereas the cell-associated virus titer in MTH52c peaked at 48 hours p.i. $\left(3.94 \times 10^{6} \mathrm{pfu} / \mathrm{well}\right)$, the maximum yield in the supernatant was observed at 96 hours p.i. $\left(1.54 \times 10^{6} \mathrm{pfu} /\right.$ well $)$. These data correlated very well with cell death and demonstrated that GLV-1h68 can efficiently replicate in MTH52c cells.

In addition, the infectivity of GLV-1h68 was assessed and compared by virus titration in two pairs of cell lines (see in Supplementary Material available online at doi $10.1155 / 2010 / 736907)$. In both cases, GLV-1h68 formed plaques 70-200 times more efficiently in cancer cells than in normal cells, indicating that GLV-1h68 preferentially replicates in tumor cells.

3.2. Confirmation of Infection and Replication of GLV-1h68 in MTH52c Cells in Cell Culture and In Vivo through Virus-Mediated Protein Expression. To verify the infection and replication of GLV-1h68 in canine carcinoma cells, we followed the expression of the virus-mediated Renilla luciferase-green fluorescent protein-fusion protein (RucGFP) and $\beta$-galactosidase (LacZ) in cell culture (Figure 2). Our Western blot analysis revealed that both marker proteins were efficiently expressed over a period of four days (Figure 2). The expression of Ruc-GFP and LacZ in cells infected at MOI of 0.1 peaked between 48 and $96 \mathrm{hpi}$, whereas maximum expression with an MOI of 1.0 was around 48 hpi.

Similar data were obtained by fluorescence microscopy (Figure 3). In these experimental settings we found that MTH52c cells infected with GLV-1h68 at MOI of 0.1 and 1.0 exhibited the strongest GFP expression at 72 and 96 hours, respectively, (supplementary Figure 1 and Figure 3). These data were also confirmed by flow cytometry (Figure 4) showing that the amount of infected cells increased over time and that those cells infected with vaccinia virus (detectable by GFP expression) were those cells that exhibited the major population of dead/dying cells (detectable by positive propidium iodide staining). In fluorescence microscopy, we used the same dye to demonstrate that most of the infected cells were dead/dying at $96 \mathrm{~h}$ p.i. (Figure 3). These results indicate that GLV-1h68 was able to efficiently infect, replicate, and kill the MTH52c cells in cell culture.

Next, we examined the efficacy of GLV-1h68 to target MTH52c tumors in vivo. For this purpose, at different days postinjection, the mice of each group were observed either under a fluorescence stereomicroscope (Leica MZ 16 FA; Wetzlar, Germany) to detect GFP-dependent fluorescence or using the low-light Imager (NightOWL LB 981, Berthold Technologies, Bad Wildbad, Germany) to detect luciferasecatalyzed light emission in the presence of intravenously injected coelenterazine (Sigma, Taufkirchen, Germany). The GFP and luciferase expressions are dependent on vaccinia 


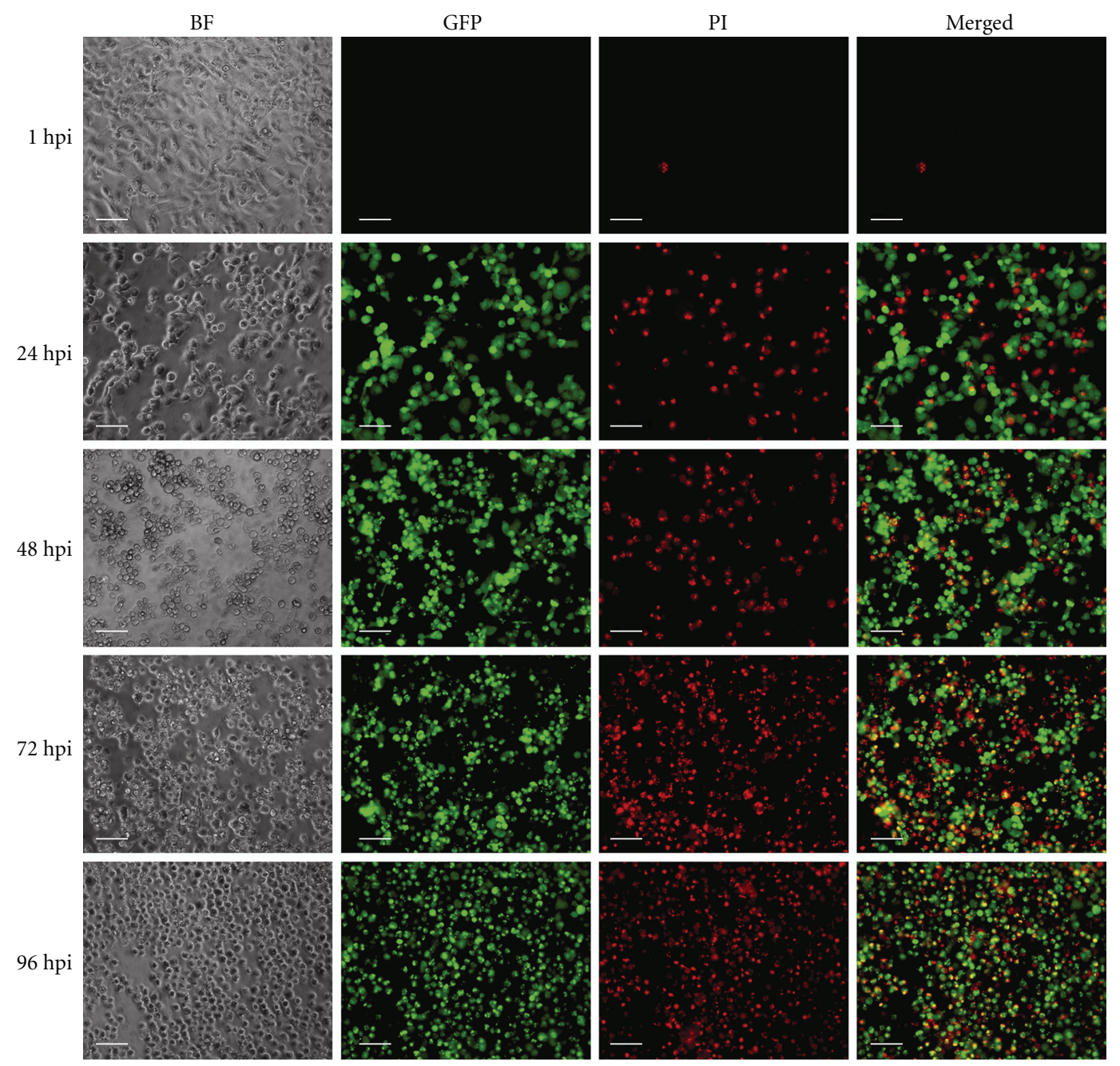

Figure 3: Time-dependent effects of infection of MTH52c with GLV-1h68 at an MOI of 1.0. (BF) Transmitted light view of virus-infected MTH52c cells; (GFP) expression of GFP in infected cells detected by direct fluorescence; (PI) propidium iodide staining of dead cells; (Merged) colocalization of GFP with the dead cells is shown in the merged imaged. All pictures in this set were taken at the same magnification. Scale bars represent $0.1 \mathrm{~mm}$.

virus replication in vivo. As demonstrated, GFP fluorescence and luminescence were detected only within tumors of GLV1h68-injected mice (Figure 5). The imaging data indicated the preferential accumulation of GLV-1h68 in MTH52c tumors.

\subsection{A Single Systemic Application of GLV-1h68 Causes Sig-} nificant Inhibition of Tumor Growth in MTH52c Xenografts. The therapeutic capacity of GLV-1h68 against an induced canine mammary cancer was tested in 10 female nude mice implanted with MTH52c cells at the age of 6-8 weeks. Twelve days postimplantation, all nude mice developed tumors with sizes between 400 and $500 \mathrm{~mm}^{3}$. Then groups of five tumorbearing mice were injected with either $5 \times 10^{6}$ pfu of GLV1 h68 or PBS (control). The tumor size of all animals was measured thrice weekly for six weeks. The single vaccinia virus injection caused an efficient inhibition of tumor growth in all GLV-1h68-treated tumor-bearing mice compared to control mice (Figure 6(a)). In addition, no reduction of net body weight of the animals was observed (Figure 6(b)).

The data revealed that GLV-1h68 could be an effective tool for the therapy of canine mammary carcinoma.

3.4. Viral Localization in GLV-1h68-Treated Mice after Inhibition of Tumor Growth. We analyzed the viral distribution in GLV-1h68-treated tumor-bearing mice by standard plaque assay and immunohistochemical staining. The plaque assay analysis revealed that viral titers in tumors were $10^{4}$ to $10^{7}$ and $10^{3}$ to $10^{5}$ times higher than the titers found in all the other organs combined at day 21 and 42, respectively (Table 1). These results show that GLV-1h68 can specifically infect and replicate in canine cancer cells.

To further examine the tumor tissues, we analyzed the tissue sections of the primary tumors at 42 days after virus injection by immunohistology. Microscopic analysis of viral distribution demonstrated that GLV-1h68 was present 
TABLE 2: Comparison of mouse immune-related protein antigen profiling in primary ZMTH3 and MTH52c tumors with or without GLV1 h68 at day 42 after virus infection $(n=2)$. Folds of enhancement (a) or suppression (b) of mouse protein expression after virus injection are shown.

(a) Protein expression level: upregulated (day 42 after virus infection).

\begin{tabular}{|c|c|c|c|}
\hline Antigen & $\begin{array}{c}\text { GLV-1h68/untreated ratio } \\
\text { (MTH52c) }\end{array}$ & $\begin{array}{c}\text { GLV-1h68/untreated ratio } \\
\text { (ZMTH3) }\end{array}$ & Classification \\
\hline Apo A1 & 7.56 & 1.21 & Anti-inflammatory protein \\
\hline IFN-gamma & 4.00 & 1.8 & Proinflammatory cytokine \\
\hline IL-6 & 7.04 & 5.52 & Proinflammatory cytokine \\
\hline IL-11 & 6.59 & 1.6 & Pleiotropic cytokine \\
\hline IL-18 & 8.00 & 3.21 & Proinflammatory cytokine \\
\hline IP-10 (CXCL10) & 29.47 & 11.21 & $\begin{array}{l}\text { Interferon-gamma-induced } \\
\text { protein }\end{array}$ \\
\hline MCP-1 (CCL2) & 18.36 & 4,99 & Proinflammatory cytokine \\
\hline MCP-3 (CCL7) & 13.26 & 2.36 & Proinflammatory cytokine \\
\hline MCP-5 (CCL12) & 3.84 & 7.13 & Proinflammatory cytokine \\
\hline $\mathrm{M}-\mathrm{CSF}(\mathrm{KC} / \mathrm{GRO} \alpha)$ & 8.28 & 3.51 & Proinflammatory cytokine \\
\hline MDC (CCL22) & 4.02 & 1.97 & chemokine \\
\hline MIP-1beta & 9.34 & 1.66 & Proinflammatory cytokine \\
\hline MIP-2 (CXCL2) & 6.91 & 11.93 & Proinflammatory chemokine \\
\hline MMP-9 & 12.68 & 26.31 & Matrix Metalloproteinase- 9 \\
\hline TIMP-1 & 5.05 & 2.91 & $\begin{array}{l}\text { Tissue inhibitor of } \\
\text { metalloproteinase type- } 1\end{array}$ \\
\hline TNF-alpha & 6.47 & 1.5 & Proinflammatory cytokine \\
\hline
\end{tabular}

(b) Protein expression level: downregulated (day 42 after virus infection).

\begin{tabular}{lccc}
\hline Antigen & $\begin{array}{c}\text { untreated/GLV-1h68ratio } \\
(\text { MTH52c) }\end{array}$ & $\begin{array}{c}\text { untreated/GLV-1h68ratio } \\
\text { (ZMTH3) }\end{array}$ & Classification \\
\hline VWF & 1.33 & 1.01 & von Willebrand factor \\
MIP-1gamma (CCL9) & 2.71 & 1.96 & Macrophage inflammatory protein \\
\hline
\end{tabular}

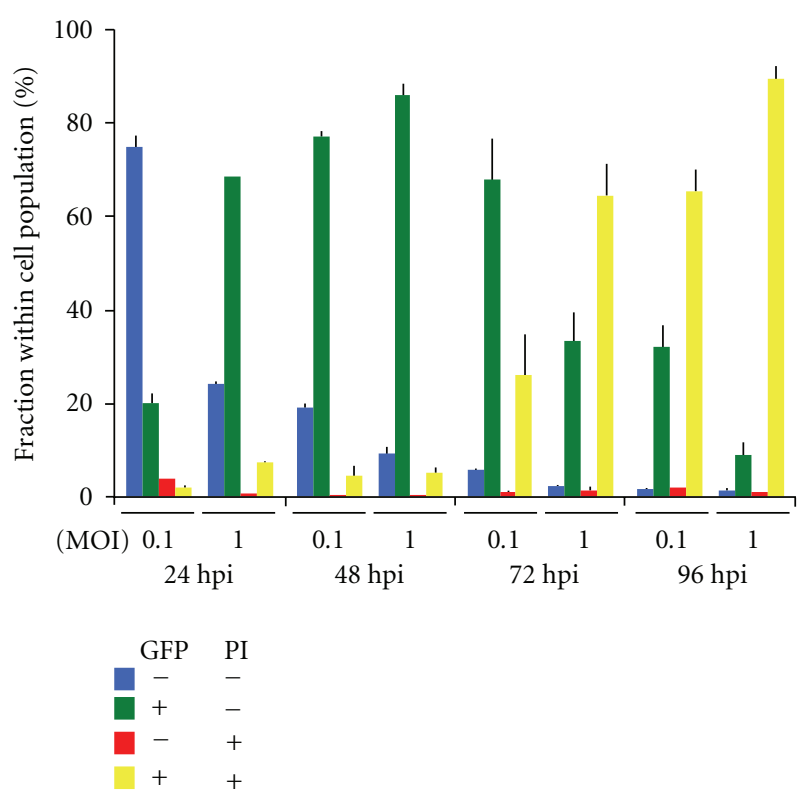

FIGURE 4: FACS analysis of MTH52c after infection with GLV-1h68 at MOIs of 0.1 and 1.0. Flow cytometry data indicate percentage of GFP and propidium iodide (PI) positive or negative cells. throughout the tumor tissue of virus-infected mice but not in control mice (Figure 7). As expected, both the virus and the GFP distribution were similar in the whole tumor tissue, indicating that in this case GFP expression is an optimal tool for the monitoring of the GLV-1h68 infection in vivo (Figure $7 \mathrm{AB}$ ). In addition, the histological data revealed that vaccinia virus infection led to oncolysis and damage of tumor tissue (Figure 7AI).

3.5. Analysis of Host Immune Response in GLV-1h68-Infected and Noninfected Primary Tumors. In order to analyze the effects of virus infection in vivo, we determined the mouse antigen profiling of GLV-1h68-infected and noninfected tumors of MTH52c or ZMTH3 xenografted nude mice. Canine mammary ZMTH3 adenoma tumors are susceptible to GLV-1h68 treatment in vivo [12] and therefore were used as an additional control.

At 42 days after virus injection, MTH52c and ZMTH3 tumors of nude mice with or without GLV-1h68 treatment were removed and used for generation of tumor tissue lysates as described in Material and Methods. 

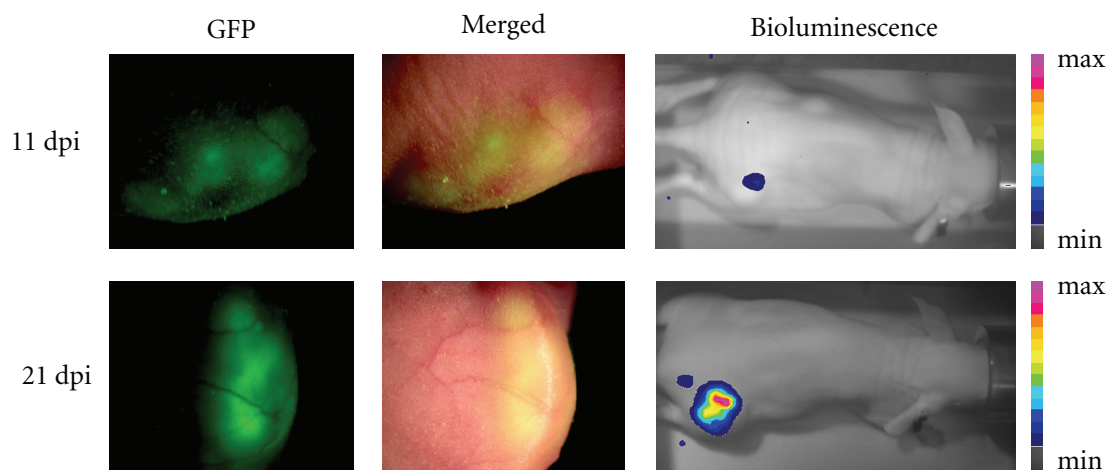

FIGURE 5: Fluorescence and luminescence imaging of MTH52c tumor-bearing mice after virus treatment. Fluorescence (GFP and Merged) imaging from the local tumor site and luminescence imaging of one representative mouse were taken 11 and 21 days post injection. Min: minimum; max: maximum.

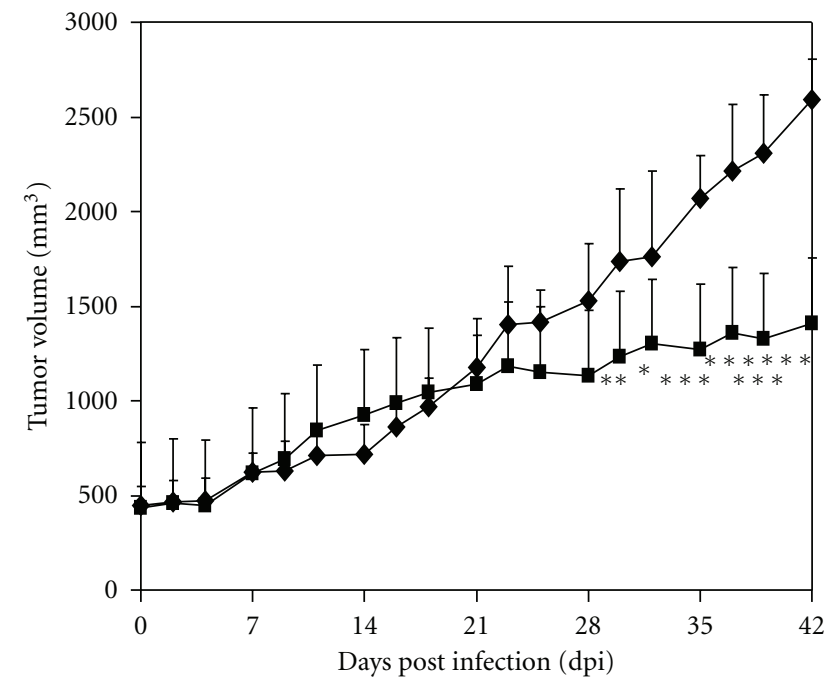

(a)

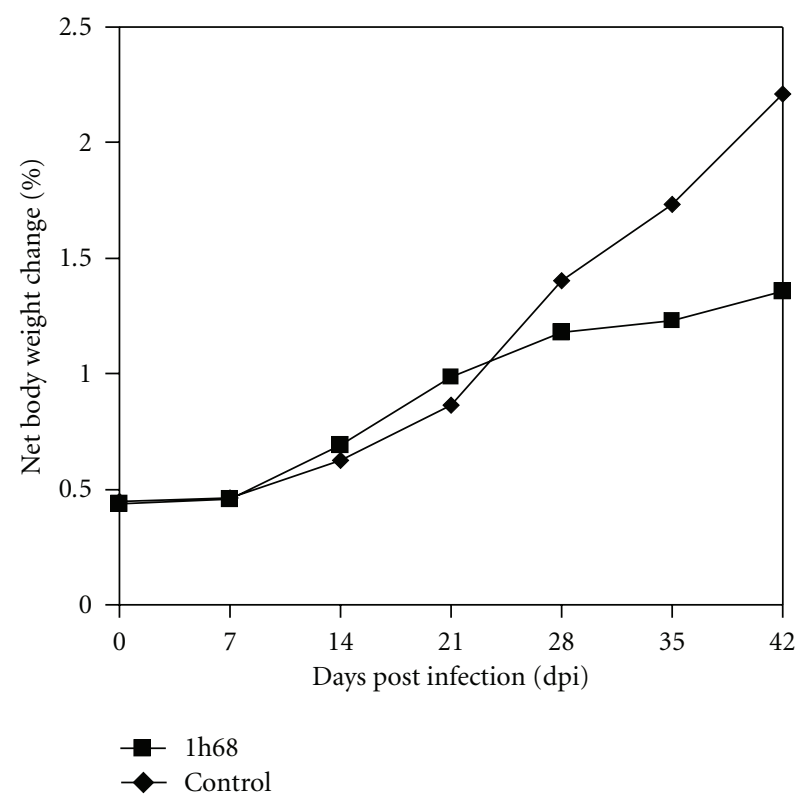

(b)

FIGURE 6: Effect of GLV-1h68 on MTH52c tumor growth in nude mice. (a) MTH52c tumor development in mice after GLV-1h68-treatment versus PBS treatment. Two-way analysis of variance (ANOVA) was used to compare the two corresponding data points of the two groups. $P<.05$ was considered as statistically significant ${ }^{*} P<.05 ;{ }^{* *} P<.01 ;{ }^{* *} P<.001$. (b) Body weights of MTH52c cell xenografted mice after virus treatment.

The data in both xenograft models revealed that GLV$1 \mathrm{~h} 68$ injection led to increased production of most of the tested proinflammatory cytokines and chemokines, such as MCP-1, MCP-3, MCP-5, M-CSF, IP-10, and IL-18 whereas only the cytokine MIP-1-gamma (CCL9) and the von Willebrand factor were downregulated (Table 2).

\section{Discussion}

Despite advances in surgery, radiation, and chemotherapy, the available treatment options for mammary carcinoma in dogs are limited and the prognosis for patients with advanced-stage disease is very poor. Therefore, the development of novel agents for therapy and diagnosis of canine mammary carcinoma is essential.

In this study, we showed for the first time that the recombinant vaccinia virus GLV-1h68 was able to effectively infect, replicate in, and lyse canine carcinoma cells in culture. The viral replication correlated well with cell lysis and with expression of the marker GLV-1h68 genes encoding $\beta$ galactosidase and Renilla luciferase-green fluorescent protein (GFP) fusion protein, respectively. In addition, flow cytometry data (Figure 4) confirmed that the virus-infected cells, detectable by GFP expression, were those cells that exhibited the major population of dead/dying cells (detectable by positive propidium iodide staining). 
$\mathrm{BF}$
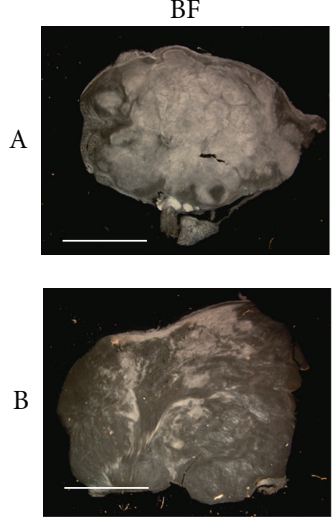

$\mathrm{BF}$

AI
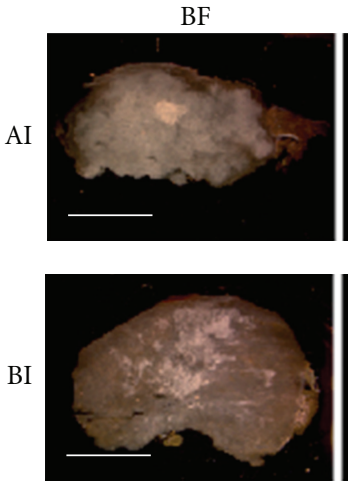

GFP
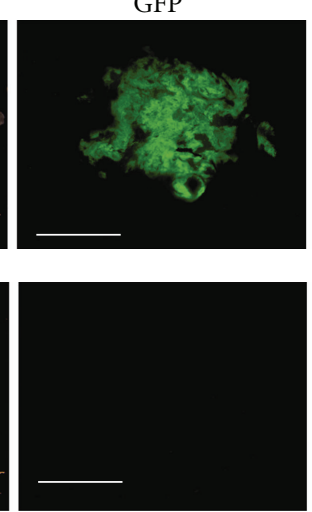

GFP
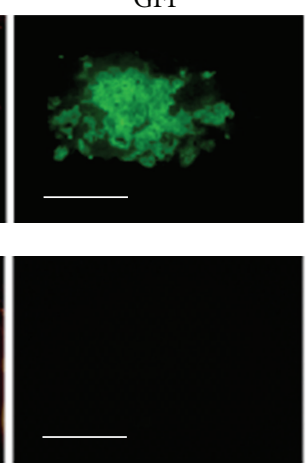

Anti-VACV
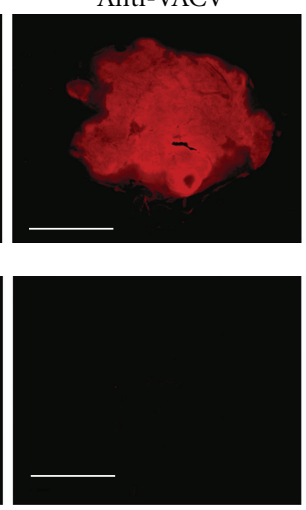

Phalloidin-TRITC
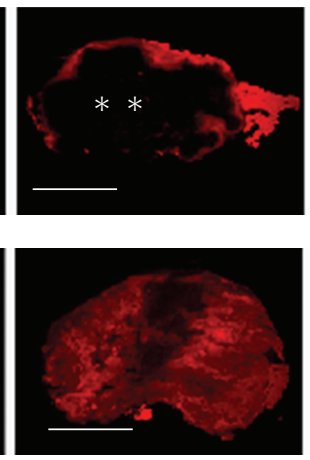
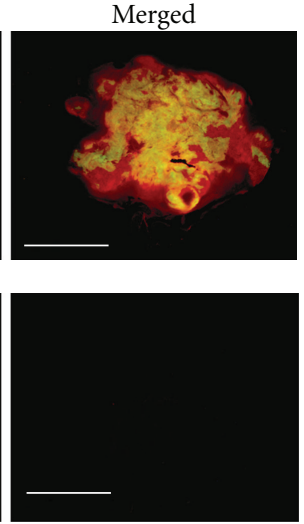

Merged
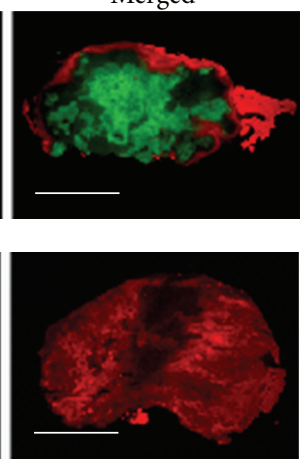

Figure 7: Immunohistochemical staining of MTH52c tumors. Tumor-bearing mice were i.v. injected either with $5 \times 10_{6}$ pfu of At day 42 after injection, GLV-1h68 (A and AI) or PBS (B and BI). whole tumor cross-sections $(100 \mu \mathrm{m})$ were labeled either with antivaccinia virus or Phalloidin-TRITC (I) antibodies (both red) and analyzed by fluorescence microscopy to detect GFP (green) and actin or vaccinia virus-dependent (red) fluorescence. Scale bars represent $5 \mathrm{~mm}$. ${ }^{*}$ Large areas lacking actin staining indicate dead tumor tissue damaged by GLV-1h68.

Taken together, we did not find any evidence of possible resistance of canine carcinoma cancer MTH52c cells to infection with vaccinia virus in cell culture.

The current study also demonstrated the ability of GLV1 h68 to provide highly effective therapy in vivo. We observed a significant inhibition of tumor growth and damage of tumor tissue in the GLV-1h68-treated tumor-bearing mice compared to control mice. Most importantly, the treated animals appeared in good health without signs of toxicity, and no reduction of net body weight of virus-infected mice was observed (Figure 6(b)). In addition, experiments analyzing viral biodistribution in different organs (Table 1), as well as GFP fluorescence and luminescence studies on the living mice (Figure 5), confirmed the fact that the GLV-1h68 virus has an outstanding infection and replication capability and specificity in tumors $[10,13]$.

In order to analyze the possible mechanism of tumor elimination by GLV-1h68 in our MTH52c tumor xenograft model, we investigated the mouse immune-related protein antigen profiling in the primary tumors with or without virus injection. In these experimental settings we also used GLV-1h68-infected tumors of ZMTH3 xenografted nude mice as an additional control. The data revealed that in both the MTH52c and the ZMTH3 virus-infected tumors, the protein expression levels of most of the tested proinflammatory cytokines and chemokines were significantly upregulated compared to the corresponding noninfected tumors (Table 2).

Many of the upregulated proteins, such as MCP-1, MCP3, MCP-5, M-CSF, IP-10, and IL-18, augment innate immunity mediated by dendritic cells, neutrophils, macrophages, and NK cells. Interestingly, similar mouse immune-related protein antigen profilings were also determinated in other xenograft models after a single GLV-1h68 injection [13, 18]. Therefore, GLV-1h68 may induce upregulation of the innate immune system, leading to increasing levels of proinflammatory cytokines. This notion is also supported by recent immunohistological studies demonstrating specific peri- and intratumoral infiltration of MHC class IIexpressing host cells (like e.g., macrophages, and dendritic cells) surrounding virus-infected cancer cells $[18,19]$. The presence of activated macrophages or dendritic cells in virusinfected xenografts only could serve as an evidence for the association between xenograft eradication and activation of the innate immune system. These findings suggest that activation of the innate immune system may act together with viral oncolysis to induce inhibition of tumor growth and tumor eradication in this model. However, which components of the innate immune system are involved in the elimination of tumor cells remains unknown.

We have reported previously that the GLV-1h68 virus can be successfully used for the treatment of canine mammary 
ZMTH3 adenoma in vivo [12]. The comparison of the antitumor effects of GLV-1h68 with that of the present study showed that, in the adenoma xenograft ZMTH3 model, GLV-1h68 injection led to a faster and more efficient tumor inhibition and regression than in mice bearing MTH52c carcinoma tumors. One possible explanation could be the better replication efficacy of GLV-1h68 in the ZMTH3 tumors compared to that of MTH52c tumors in vivo [12]. However, a significant inhibition of the tumor growth was found in both ZMTH3 and MTH52c xenografts at day 30 after virus injection.

Therefore, GLV-1h68 could be a useful tool for treatment of both mammary cancer types in canine patients.

\section{Conclusion}

Our study demonstrates that the attenuated vaccinia virus strain GLV-1h68 can efficiently infect and destroy the canine mammary carcinoma MTH52c cells in cell culture and in vivo. In addition, a single systemic administration of GLV-1h68 causes a significant inhibition of tumor growth in MTH52c xenografts and damage of tumor tissue without detectable effects on the health status of the treated animals.

In summary, these data indicate that GLV-1h68 is a promising candidate virus in the treatment of breast carcinomas in canine patients.

\section{Abbreviations}

$\begin{array}{ll}\text { ApoA1: } & \text { Apolipoprotein A1 } \\ \text { IL-6: } & \text { Interleukin-6 } \\ \text { IP-10: } & \text { Interferon-inducible protein } \\ \text { MCP-1: } & \text { Monocyte chemoattractant protein-1 } \\ \text { M-CSF: } & \text { Macrophage colony-stimulating factor } \\ \text { MIP: } & \text { Macrophage inflammatory protein } \\ \text { MMP-9: } & \text { Matrix metalloproteinase 9 } \\ \text { MOI: } & \text { Multiplicities of infection } \\ \text { TIMP-1: } & \text { Metallopeptidase inhibitor 1 } \\ \text { TNF-alpha: } & \text { Tumor necrosis factor-alpha } \\ \text { VWF: } & \text { Von Willebrand factor. }\end{array}$

\section{Acknowledgments}

The authors declare that they have competing interests. I. Gentschev, N. Chen, Y. A. Yu, Q. Zhang, J. Stritzker, and A. A. Szalay have financial interests in Genelux Corporation. K. Ehrig, M. Hess, S. Rudolph, and U. Donat were supported by grants of Genelux Corporation. The costs of publication of this paper were defrayed in part by the payment of page charges. This paper must therefore be hereby marked advertisement in accordance with 18 U.S.C. Section 1734 solely to indicate this fact. The authors thank Ms. J. Langbein for excellent technical support and Dr. D. Haddad and Dr. Z. Sokolovic for critical reading of the paper. I. Gentschev and K. Ehrig contributed equally to this paper.

\section{References}

[1] M. Mottolese, L. Morelli, U. Agrimi et al., "Spontaneous canine mammary tumors: a model for monoclonal antibody diagnosis and treatment of human breast cancer," Laboratory Investigation, vol. 71, no. 2, pp. 182-187, 1994.

[2] E. Hellmén, "Complex mammary tumours in the female dog: a review," Journal of Dairy Research, vol. 72, pp. 90-97, 2005.

[3] K. Sorenmo, "Canine mammary gland tumors," Veterinary Clinics of North America-Small Animal Practice, vol. 33, no. 3, pp. 573-596, 2003.

[4] M. J. V. Vähä-Koskela, J. E. Heikkilä, and A. E. Hinkkanen, "Oncolytic viruses in cancer therapy," Cancer Letters, vol. 254, no. 2, pp. 178-216, 2007.

[5] A. M. Crompton and D. H. Kirn, "From ONYX-015 to armed vaccinia viruses: the education and evolution of oncolytic virus development," Current Cancer Drug Targets, vol. 7, no. 2, pp. 133-139, 2007.

[6] T.-C. Liu, E. Galanis, and D. Kirn, "Clinical trial results with oncolytic virotherapy: a century of promise, a decade of progress," Nature Clinical Practice Oncology, vol. 4, no. 2, pp. 101-117, 2007.

[7] R. Cattaneo, T. Miest, E. V. Shashkova, and M. A. Barry, "Reprogrammed viruses as cancer therapeutics: targeted, armed and shielded," Nature Reviews Microbiology, vol. 6, no. 7, pp. 529-540, 2008.

[8] D. H. Kirn and S. H. Thorne, "Targeted and armed oncolytic poxviruses: a novel multi-mechanistic therapeutic class for cancer," Nature Reviews Cancer, vol. 9, no. 1, pp. 64-71, 2009.

[9] A. Worschech, D. Haddad, D. F. Stroncek, E. Wang, F. M. Marincola, and A. A. Szalay, "The immunologic aspects of poxvirus oncolytic therapy," Cancer Immunology, Immunotherapy, vol. 58, no. 9, pp. 1355-1362, 2009.

[10] Q. Zhang, Y. A. Yu, E. Wang et al., "Eradication of solid human breast tumors in nude mice with an intravenously injected light-emitting oncolytic vaccinia virus," Cancer Research, vol. 67, no. 20, pp. 10038-10046, 2007.

[11] K. J. Kelly, Y. Woo, P. Brader et al., "Novel oncolytic agent GLV1 h68 is effective against malignant pleural mesothelioma," Human Gene Therapy, vol. 19, no. 8, pp. 774-782, 2008.

[12] I. Gentschev, J. Stritzker, E. Hofmann et al., "Use of an oncolytic vaccinia virus for the treatment of canine breast cancer in nude mice: preclinical development of a therapeutic agent," Cancer Gene Therapy, vol. 16, no. 4, pp. 320-328, 2009.

[13] Y. A. Yu, C. Galanis, Y. Woo et al., "Regression of human pancreatic tumor xenografts in mice after a single systemic injection of recombinant vaccinia virus GLV-1h68," Molecular Cancer Therapeutics, vol. 8, no. 1, pp. 141-151, 2009.

[14] S.-F. Lin, D. L. Price, C.-H. Chen et al., "Oncolytic vaccinia virotherapy of anaplastic thyroid cancer in vivo," Journal of Clinical Endocrinology and Metabolism, vol. 93, no. 11, pp. 4403-4407, 2008.

[15] S.-F. Lin, Z. Yu, C. Riedl et al., "Treatment of anaplastic thyroid carcinoma in vitro with a mutant vaccinia virus," Surgery, vol. 142, no. 6, pp. 976-983, 2007.

[16] K. A. Sterenczak, S. Willenbrock, M. Barann et al., "Cloning, characterisation, and comparative quantitative expression analyses of receptor for advanced glycation end products (RAGE) transcript forms," Gene, vol. 434, no. 1-2, pp. 35-42, 2009.

[17] S. Weibel, J. Stritzker, M. Eck, W. Goebel, and A. A. Szalay, "Colonization of experimental murine breast tumours by 
Escherichia coli K-12 significantly alters the tumour microenvironment," Cellular Microbiology, vol. 10, no. 6, pp. 12351248, 2008.

[18] I. Gentschev, U. Donat, E. Homann, et al., "Regression of human prostate tumors and metastases in nude mice following treatment with the recombinant oncolytic vaccinia virus GLV-1h68," Journal of Biomedicine and Biotechnology, vol. 2010, Article ID 489759, 11 pages, 2010.

[19] A. Worschech, N. Chen, Y. A. Yu et al., "Systemic treatment of xenografts with vaccinia virus GLV-1h68 reveals the immunologic facet of oncolytic therapy," BMC Genomics, vol. 10, article 301, 2009. 


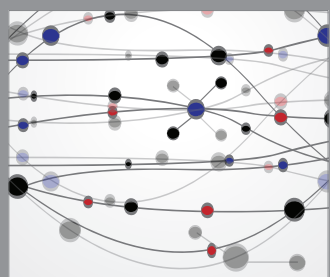

The Scientific World Journal
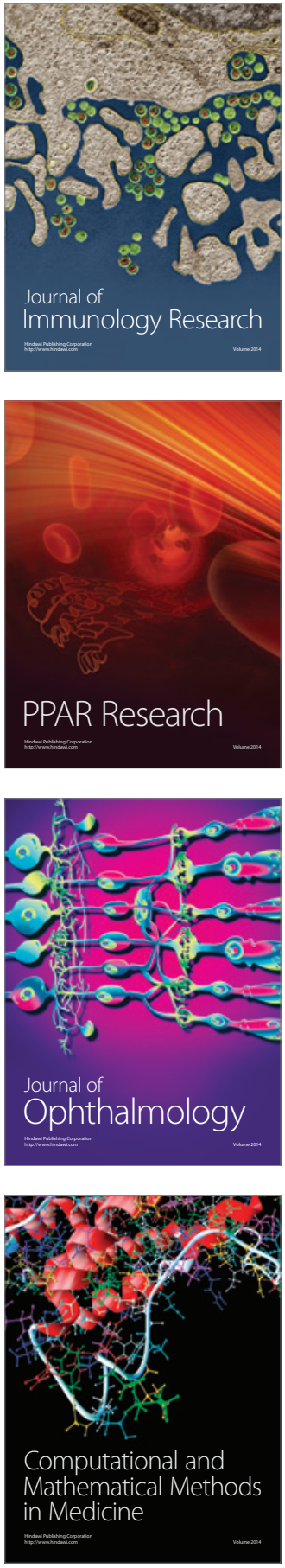

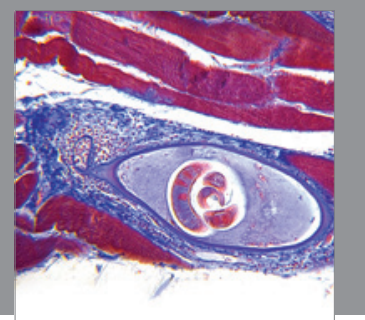

Gastroenterology

Research and Practice
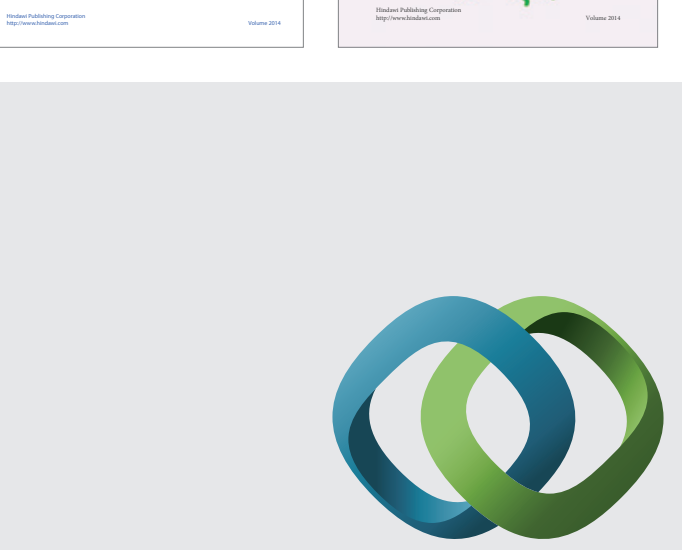

\section{Hindawi}

Submit your manuscripts at

http://www.hindawi.com
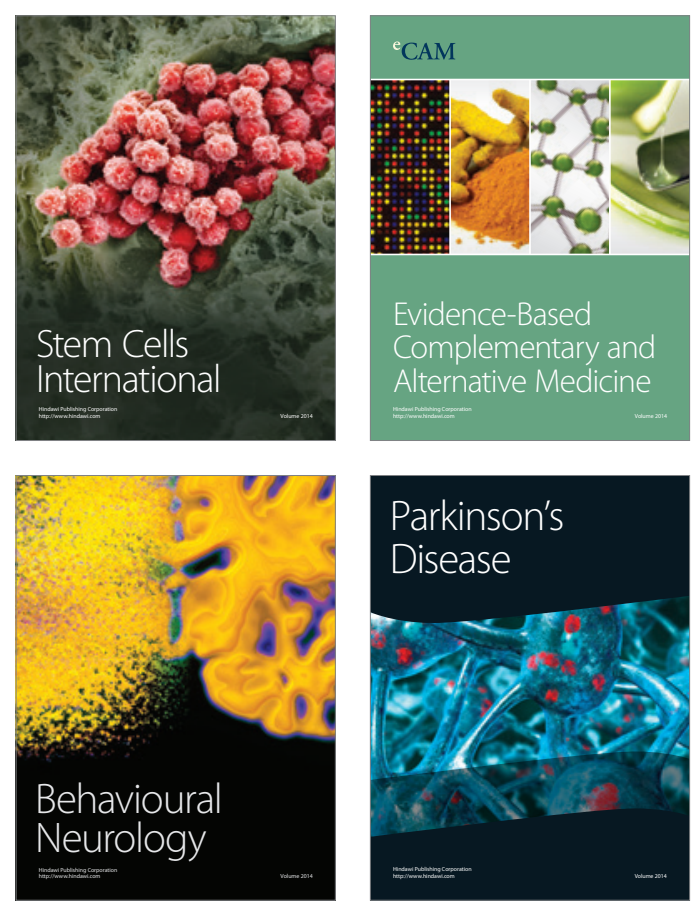

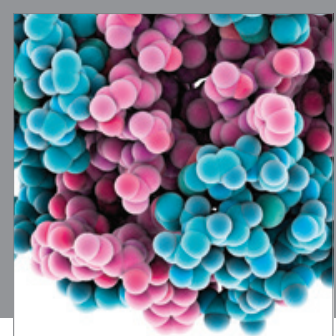

Journal of
Diabetes Research

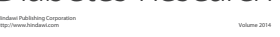

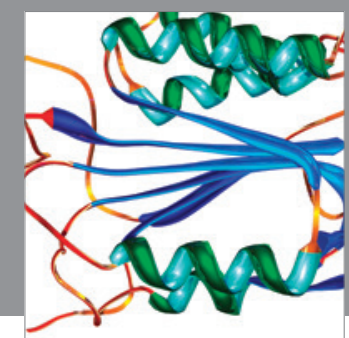

Disease Markers
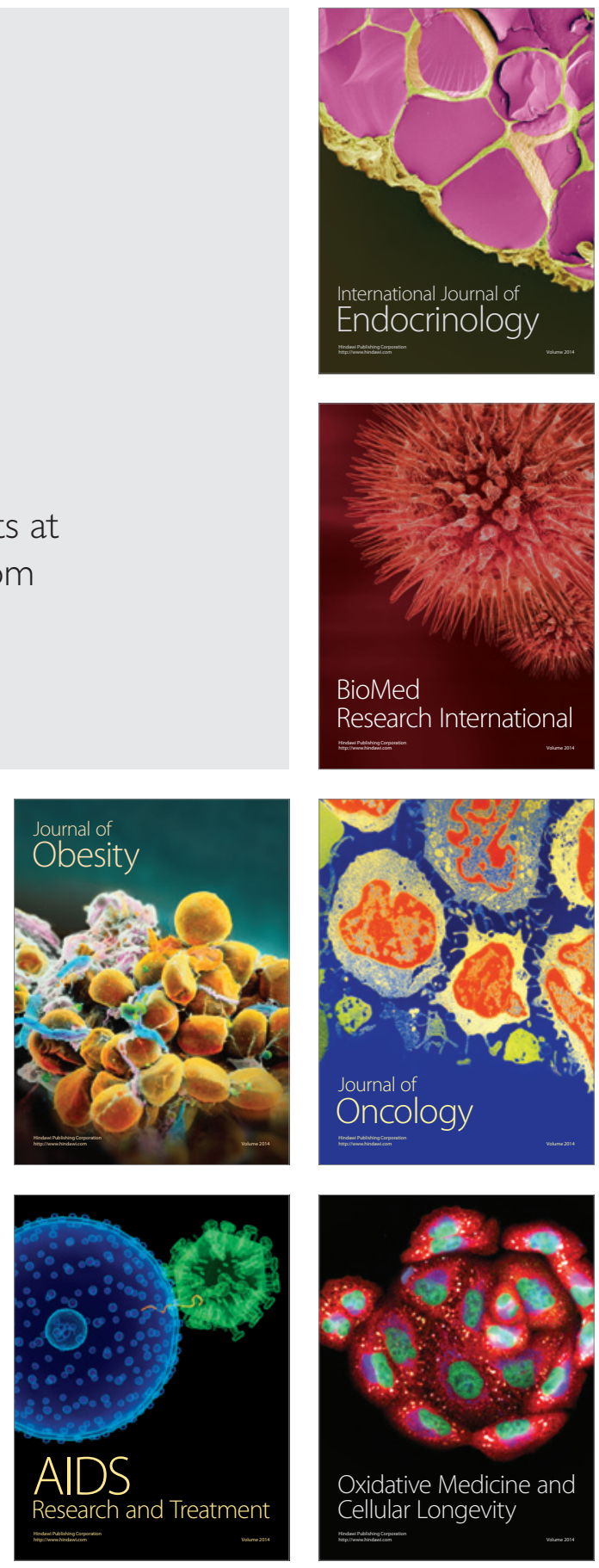